\title{
Mortality Rate and Case Fatality Rate of COVID-19 Around The World: An Adjusted Estimation
}

\section{Moslem Taheri Soodejani}

Shahid Sadoughi University of Medical Sciences and Health Services

\section{Ali Akbar Haghdoost}

Kerman University of Medical Sciences

\section{Mohammad Hassan Lotfi}

Shahid Sadoughi University of Medical Sciences and Health Services

\section{Marzieh Mahmudimanesh}

Kerman University of Medical Sciences

Seyyed Mohammad Tabatabaei ( $\sim$ moh.taba@gmail.com )

Mashhad University of Medical Sciences https://orcid.org/0000-0002-3153-8968

\section{Research}

Keywords: Coronavirus, SARS-COV-2, Case Fatality Rate, Mortality

Posted Date: January 21st, 2021

DOI: https://doi.org/10.21203/rs.3.rs-151186/v1

License: (c) (1) This work is licensed under a Creative Commons Attribution 4.0 International License. Read Full License 


\section{Mortality Rate and Case Fatality Rate of COVID-19 around the World: An Adjusted Estimation}

Moslem Taheri-Soodejani ${ }^{1}$, Ali-Akbar Haghdoost ${ }^{2}$, Mohammad-Hassan Lotfi ${ }^{1}$, Marzieh

Mahmudimanesh ${ }^{3}$, Seyyed-Mohammad Tabatabaei ${ }^{4,5 *}$

1- Research Center of Prevention and Epidemiology of Non-Communicable Disease, Department of Biostatistics and Epidemiology, Shahid Sadoughi University of Medical Sciences, Yazd, Iran

2- HIV/STI Surveillance Research Center, WHO Collaborating Center for HIV Surveillance, Institute for Futures Studies in Health, Kerman University of Medical Sciences, Kerman, Iran

3- $\quad$ PhD student in Biostatistics, Department of Epidemiology and Biostatistics, School of Health, Kerman University of Medical Sciences, Kerman, Iran

4- Medical Informatics Department, School of Medicine, Mashhad University of Medical Sciences, Mashhad, Iran

5- Clinical Research Unit, Imam Reza Hospital, Mashhad University of Medical Sciences, Mashhad, Iran

*Corresponding Author: Seyyed Mohammad Tabatabaei

Email: sm.tabatabaei@sbmu.ac.ir 


\section{Abstract}

Background: The present study is designed to predict the global adjusted values for mortality rate and case fatality rate of COVID-19 around the world.

Methods: This research was conducted at the ecological level using data from 100 countries which were chosen randomly. The adjusted values were predicted using beta regression considering predictive factors such as total expenditure on health per capita, expenditure on health as a percentage of GDP, life expectancy and the percentage of the population aged over 65 years, hospital beds (per 1000 population), physicians (per 1000 population), nurses (per 1000 population), prevalence of smoking, prevalence of diabetes mellitus, and number of confirmed tests in each country. In the end, applying Monte Carlo simulation, the adjusted values of mortality rate and case fatality rate for the whole world were estimated.

Results: The results of this study showed that two factors including percentage of population ages 65 and above $(\mathrm{P}=0.03)$ and Total expenditure on health as $\%$ of GDP $(\mathrm{P}=0.04)$ had a statistically significant relationship with the case fatality rate. Moreover, there was a statistically significant relationship between the mortality rate and life expectancy $(\mathrm{P}=0.02)$, total expenditure on health per capita ( $\mathrm{P}<0.001)$, nurses (Per 1000 Population) $(\mathrm{P}=0.04)$, and the prevalence of Diabetes Mellitus $(\mathrm{P}=0.04)$. The mortality rate and case fatality rate for the whole world were estimated to be 0.000001 and 0.026 , respectively.

Conclusion: It seems that what can cause global concern is not the case fatality rate of the disease, but its mortality rate, which is directly related to the health status of a community. The worse the health status of a community, the greater the number of infected people likely to be there, that ultimately increases the mortality rate of the disease in the community.

Keywords: Coronavirus, SARS-COV-2, Case Fatality Rate, Mortality 


\section{Background}

According to the World Health Organization, almost all countries in the world are currently infected with COVID-19. Although studies have shown that about $85 \%$ of people with the disease are asymptomatic or have mild symptoms, and only $15 \%$ have serious conditions $(10 \%$, with a case fatality rate of $15 \%)$ and critical $(5 \%$, with a case fatality rate of $50 \%)$ [1].

Case fatality rate and mortality rate are two indices used to evaluate the severe consequences of a disease. At the onset of the 2020 pandemic, known as the COVID-19, several studies have been conducted to assess these two indices [2, 3].

In every health system, there are two important indices which can be affected by various individual or public factors. Patient's age is a factor that may increase the case fatality rate of a disease. A study on deaths due to COVID-19 in Italy found that a significant proportion of deaths occurred in patients over 65 years of age [4].

Life expectancy at birth is considered as an index to assess the health status in a community. In addition, Total expenditure on health as a percentage of GDP and total expenditure on health per capita are the other indices that may have an impact on the assessment of the health status in communities and also may affect the mortality rate [5-7]. Other indices representing the health status in a country include hospital bed (per 1000 Population), physician (per 1000 Population), nurse (per 1000 Population), prevalence of smoking and prevalence of diabetes mellitus. They can affect the mortality rate either directly or indirectly [8-10].

This study aims to investigate some of the predictive factors which may affect the mortality rate and case fatality rate in 100 randomly chosen countries in order to predict the adjusted values of these two indices for each one and estimate them for the whole world.

\section{Methods}

In other to estimate case fatality rate which is defined as the proportion of deaths caused by the disease compared to the total number of cases diagnosed with it for a certain period of time [1] and mortality rate which is proportion of death by the disease to the total population for a certain period of time [2] for COVID-19 
around the world, data from 100 countries which were chosen randomly. Reports from World Health Organization, World Bank and World Meters were the resources to collect the required data-including the number of confirmed cases, the number of diagnostic tests per 1 million people, the number of deaths due to COVID-19, total population, life expectancy, total expenditure on health as $\%$ of GDP, total expenditure on health per capita $(\$)$ and the percentage of the population aged over 65 years in each country one by one.

Beta regression was chosen to explore the effect of predictive factors on predicting two indices of case fatality rate and mortality rate. This analysis is used in estimates where the response variable takes values between zero and one. One of the distributions with a variable ranging from zero to one is beta distribution. In this regression model, the density function of the response variable uses its other form $(y \sim \mathcal{B}(\mu, \varphi))$ instead of the usual beta distribution form $(y \sim \mathcal{B}(a, b))$ where $\mu=\frac{a}{a+b}$ and $\varphi=a+b$. The beta regression model is then defined as follows:

$g\left(\mu_{i}\right)=x_{i}^{T} \beta_{i}$

Where $\beta=\left(\beta_{1}, \ldots, \beta_{k}\right)^{T}$ is a $k \times 1$ vector which is actually the vector of the regression parameters, $x_{i}=\left(x_{i 1}, \ldots, x_{i k}\right)^{T}$ is a vector including $\mathrm{k}$ independent variable, and $g():.(0,1) \mapsto \mathbb{R}$ is a link function. The most widely used link function in this regression model is the log function, which is defined as $g(\mu)=$ $\log \left(\frac{\mu}{(1-\mu)}\right)$. The present study, too, makes use of this form [11].

After estimating the coefficients for each variable by beta-regression, mortality rate and case fatality rate estimation were conducted for every country using statistically significant coefficients. According to these predictions, three clusters were defined using a dendrogram at first. Then, the countries were divided into three clusters based on their case fatality rate and mortality rates using K-Means clustering method - a non-hierarchical method. Countries with the greatest values were considered as high, countries with the lowest values were considered as low, and countries with the values between these two were considered as moderate. In K-Means clustering method, each observation is assigned to a single cluster with the closest central mean when a Euclidean distance is used to measure the distance between an observation and the mean. 
Afterwards, case fatality rate and mortality rate for the whole world was estimated using the Monte Carlo simulation method in which the bootstrap method estimates case fatality rate and mortality rate for all countries under investigation. the percentages of 2.5 and 97.5 were determined for them defining the lower and the upper limit of the confidence interval [12].

All of the data were extracted from World Bank, World Meters and WHO websites on 14th, May. Data were analyzed using RStudio Version 1.2.5001.

\section{Results}

The assessment of some predictive factors affecting mortality rate and case fatality rate revealed that life expectancy $(\mathrm{P}=0.02)$, total expenditure on health per capita ( $\mathrm{P}<0.001)$, nurses (Per 1000 Population) $(\mathrm{P}=0.04)$, and the prevalence of Diabetes Mellitus $(\mathrm{P}=0.04)$ were statistically significant and had an impact on the mortality rate of SARS-COV-2 in a community. However, total expenditure on health as $\%$ of GDP and the percentage of the population aged over 65 years, hospital beds (per 1000 population), physicians (per 1000 population), and prevalence of smoking were not statistically significant.

The results of the analyzes performed on the predictive factors affecting the case fatality rate of SARS-COV-2 showed that only the percentage of the population aged over 65 years $(\mathrm{P}=0.03)$ and Total expenditure on health as $\%$ of GDP $(\mathrm{P}=$ 0.04 ) were statistically significant, but other examined variables were found not to be statistically significant. The number of diagnostic tests performed in each country to identify confirmed cases of COVID-19 was entered to the model as an adjusting variable. More details are listed in Table 1.

Table 1: Predictive factors associated with mortality rate and case fatality rate

\begin{tabular}{|c|c|c|c|c|}
\hline \multirow{2}{*}{ Predictive Factors } & \multicolumn{2}{|l|}{ Mortality Rate* } & \multicolumn{2}{|l|}{ Case Fatality Rate** } \\
\hline & Coefficient ( $95 \% \mathrm{CI})$ & $\mathrm{P}$ & Coefficient $(95 \% \mathrm{CI})$ & $\mathrm{P}$ \\
\hline Life Expectancy & $-0.05(-0.1 ;-0.006)$ & 0.02 & $-0.004(-0.034 ; 0.026)$ & 0.79 \\
\hline $\begin{array}{l}\text { Total expenditure on } \\
\text { health as \% of GDP }\end{array}$ & $0.041(-0.05 ; 0.14)$ & 0.4 & $0.059(0.001 ; 0.11)$ & 0.04 \\
\hline
\end{tabular}




\begin{tabular}{|l|l|l|l|l|}
\hline $\begin{array}{l}\text { Total expenditure on } \\
\text { health per capita(\$) }\end{array}$ & $0.0003(0.0001 ; 0.0005)$ & $<0.001$ & $-0.00003(-0.0001 ; 0.00009)$ & 0.62 \\
\hline$\%$ up 65 Year & $0.04(-0.01 ; 0.11)$ & 0.14 & $0.039(0.002 ; 0.07)$ & 0.03 \\
\hline $\begin{array}{l}\text { Hospital Beds(Per } \\
1000 \text { Population) }\end{array}$ & $-0.01(-0.11 ; 0.09)$ & 0.8 & $-0.05(-0.12 ;-0.01)$ & 0.1 \\
\hline $\begin{array}{l}\text { Physicians (Per 1000 } \\
\text { Population) }\end{array}$ & $-0.06(-0.24 ; 0.12)$ & 0.52 & $-0.012(-0.12 ; 0.09)$ & 0.8 \\
\hline $\begin{array}{l}\text { Nurses (Per 1000 } \\
\text { Population) }\end{array}$ & $-0.01(-0.19 ;-0.002)$ & 0.04 & $-0.02(-0.07 ; 0.02)$ & 0.37 \\
\hline Smoking (Prevalence) & $0.007(-0.2 ; .03)$ & 0.6 & $0.0006(-0.015 ; 0.016)$ & 0.93 \\
\hline $\begin{array}{l}\text { Diabetes Mellitus } \\
\text { (Prevalence) }\end{array}$ & $0.06(0.01 ; 0.12)$ & 0.04 & $0.004(-0.035 ; 0.044)$ & 0.82 \\
\hline $\begin{array}{l}\text { Confirming Test (Per 1 } \\
\text { Million Population) }\end{array}$ & $\begin{array}{l}-0.0000005(-000001 \\
0.00000004)\end{array}$ & 0.07 & $\begin{array}{l}-0.0000007(-0.000001 ; \\
0.0000001)\end{array}$ & $<0.01$ \\
\hline
\end{tabular}

*Mont Carlo Simulation for world: $0.000001(0 ; 000003)$

**Mont Carlo Simulation for world: $0.026(0.012 ; 0.041)$

Using statistically significant variables, the mortality rate and case fatality rate for each country were predicted, and based on the Euclidean distance; these countries were divided into three clusters with low, moderate and high overall rate.

The clustering results showed that some countries such as Germany, Netherlands are close neighbors and they were at a same status in terms of mortality rate and the case fatality rate and their status was red but although both countries are neighbors of Belgium, the status of Belgium was different and it was put in "moderate" cluster which is colored as yellow in figure 1. Luxembourg borders Germany, Belgium and France but its status was different from them. It was put in "low" cluster while Germany and France were put in "high" cluster and Belgium in "moderate" cluster.

It was the same in other European countries, for example Spain and Portugal are neighbors but they had different status. Denmark and Germany, despite being neighbors, were put in two completely different clusters of "low" and "high". 
Sweden and Finland were put in the "high" cluster, but Norway which is in the neighborhood of these countries, was put in the "moderate" cluster.

Finland, Estonia, Latvia, Belarus, Ukraine are in the neighborhood of Russia; but the status of Russia was different from these countries in terms of mortality rate and case fatality rate.

Although Canada and United states are neighbors but they were put in different clusters.

Israel and Lebanon are close neighbors but they had different status. Also Saudi Arabia and Kuwait were at a same status but the status of Iraq which is their neighbor was different (Fig. 1).

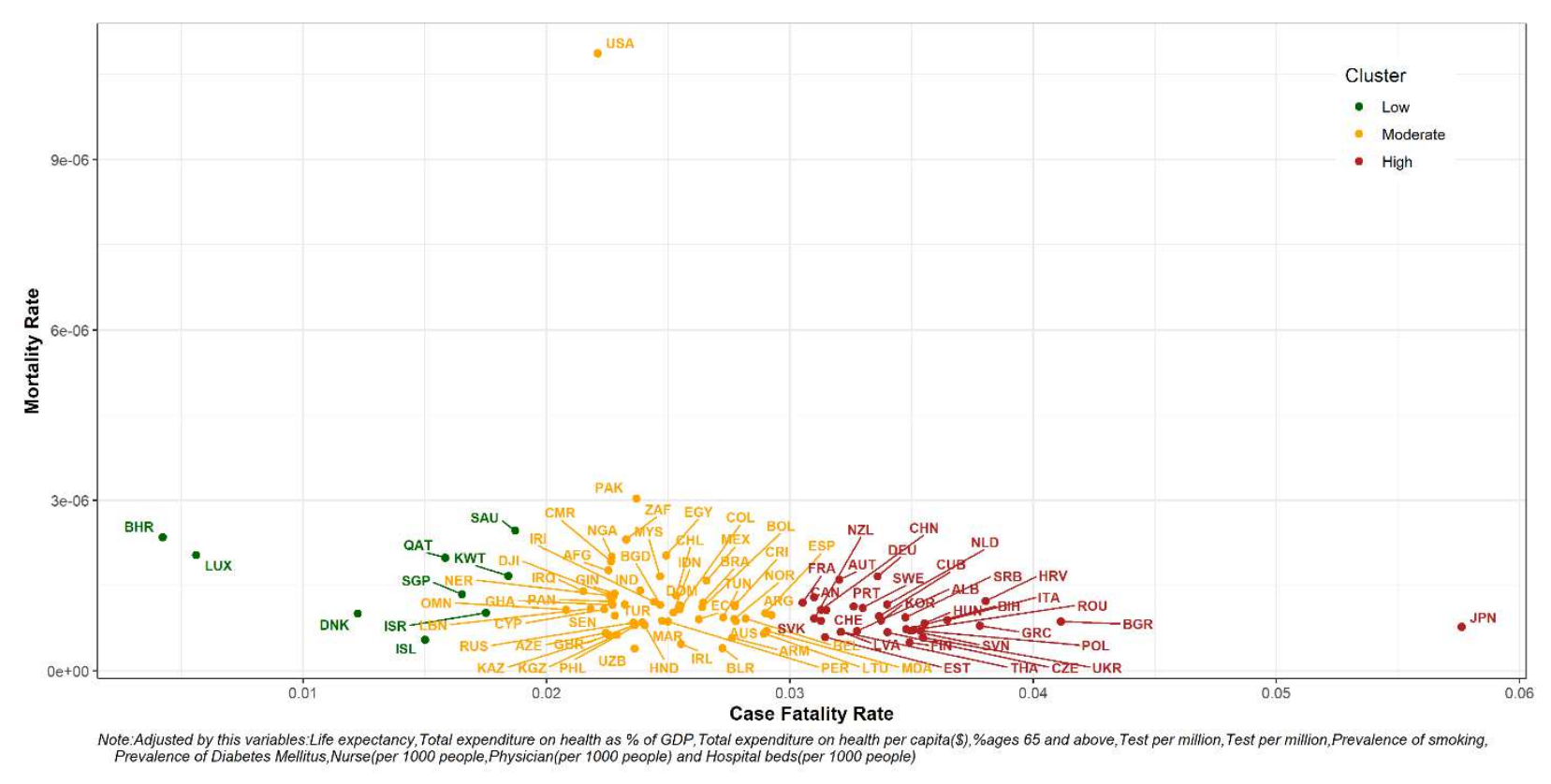

Figure 1: Country-based clustering due to the low, medium and high rate of adjusted mortality rate and case fatality rate.

The Monte Carlo simulation performed on the predicted values for each country, showed that the adjusted mortality rate and case fatality rate for the world were 0.000001 and 0.026 , respectively.

\section{Discussion}


Analyzing the predictive in order to predict case fatality rate proved that total expenditure on health as \% of GDP and the percentage of the population aged over 65 years are statistically significant. Age over 65 years has been considered as one of the predictive factors for the case fatality rate of this virus [13]. For example, Germany which is one of the aged European countries, had a higher mortality rate than the others since the highest percentage of deaths occurred in people aged over 60 [2].

The number of hospital beds is also one of the indices representing health status with an impact on the case fatality rate. Obviously, larger number of hospital beds per population would result in better hospitalization and care provision of acute cases of the disease. [8-10].

It seems that other factors such as life expectancy, hospital beds (Per 1000 Population), physicians (per 1000 population), nurses (per 1000 population), prevalence of smoking, prevalence of diabetes mellitus, and total expenditure on health per capita did not have a significant impact on this index in different countries and the only effective factors in predicting it were age distribution, total expenditure on health as \% of GDP and confirming test (per a million population).

The results indicated that life expectancy, which is affected by deaths at young ages, total expenditure on health per capita $(\mathrm{P}=0.001)$, nurses (per 1000 population) and the prevalence of diabetes mellitus were the four factors with a statistically significant impact on the mortality rate and were used in predicting the mortality rate for countries, unlike the case fatality rate. These factors are among the most important indices in every health system which represents the health status of community, so their increase is accompanied by the decrease of mortality rate in that community [14] .On the other hand, expenditure on health per capita has a strong relationship with health outcomes, therefore its increase is accompanied by the improvement of health outcomes in a community $[5,7]$.

Members of clusters which are the countries, represented different mortality patterns, even those with common geographical borders. Luxembourg and Germany are two neighboring countries, but Luxembourg had a better condition in terms of mortality due to COVID-19. This difference seems to be related to the 
facts that Luxembourg has a younger population, more tests per 1 million, and also lower prevalence of Diabetes Mellitus than Germany. [15-17]

Denmark and Germany also, despite being neighbors, had different situations since Denmark had a better condition in terms of COVID-19 mortality rate. Denmark has a lower prevalence of smoking and also a lower prevalence of Diabetes Mellitus than Germany and this seems to be the cause of this difference $[15,16]$.

Belgium and the Netherlands were other European neighbors that had different conditions in terms of mortality rate of COVID-19. Belgium has a younger population, better total expenditure on health per capita, and also more hospital beds per population, all of which can justify its lower mortality rate compared to the Netherlands [15-17].

Norway and Sweden, despite being so close to each other, fell into two different clusters. Norway was in a relatively better situation, which could be because of having a younger population, more hospital beds per population, and also better total expenditure on health per capita than Sweden [15-17].

United States and Canada are neighbors, but they had different situation in terms of COVID-19 mortality rate as United States was in a relatively better situation and having a younger population could be the reason [17].

Russia shares borders with Finland, Estonia, Latvia, Belarus and Ukraine, but it had a relatively better condition than the other five, which may be due to having a younger population, higher total expenditure on health per capita (\$) and greater number of physicians compared to the five neighbors [15-17].

Although Israel and Lebanon are neighbors, Israel is in a better situation. It seems logical since in Israel, the number of nurses and doctors, life expectancy, total expenditure on health per capita (\$) and the number of tests per 1 million in Israel is superior compared to Lebanon and also there is a lower prevalence of diabetes and smoking in Israel than in Lebanon $[15,16]$.

Moreover, there was a difference between two other neighboring countries, Saudi Arabia and Iraq. Saudi Arabia was in a better situation than Iraq and it seems that the reasons might be the greater number of physicians and nurses (per 1000 people), lower prevalence of Diabetes Mellitus, higher total expenditure on health 
per capita(\$) and greater number of tests per 1 million in Saudi Arabia than Iraq [17].

The results of Monte Carlo simulation showed that the case fatality rate was equal to its raw value. This could be due to the fact that, generally, the investigated factors such as manpower, equipment and infrastructure, do not have much effect on the fatality of the virus and if there is a regional difference, it could be caused by other reasons such as genetic characteristics of the community members. On the other hand, in the case of mortality rate, it is different since the Monte Carlo simulation showed that the mortality rate for the world is much less than its raw value, and this demonstrates that the mortality rate can be affected by factors such as the prevalence of underlying diseases, equipment and infrastructure.

\section{Conclusions}

It seems that what we all need to be worried about, at global level is not the case fatality rate of this disease, but the mortality rate, which is of course directly related to the health status of a community. Poor health status of a community would lead to an increase in the number of infected cases and as a result in the mortality rate of the disease.

\section{Limitations of the study}

This study was conducted ecologically and its results cannot be interpreted individually. Besides, many factors may have an impact on the mortality rate and case fatality rate of a disease; in this study, however, only a few important factors were investigated. Also it should be mentioned that this study can be affected with underreporting of deaths.

\section{List of abbreviations}

GDP: Gross Domestic Product 


\section{Declarations}

Ethics approval and consent to participate

Not applicable.

Consent for publication

Not applicable.

Availability of data and materials

The dataset are available from the corresponding author on reasonable request.

\section{Competing interests}

The authors declare that they have no competing interests.

\section{Funding}

None.

\section{Authors' contributions}

MTS and AAH designed the study. MHL and MMM and SMT analyzed and interpreted the Data. MTS and SMT prepared the draft. SMT reviewed and revised the manuscript. All authors read and approved the final manuscript.

\section{Acknowledgements}

Not applicable 


\section{References}

1.Taheri Soodejani M, Lotfi MH, Tabatabaei SM. Is case fatality rate an appropriate index to represent the status of case-finding process for COVID-19 in different countries? Infection Ecology \& Epidemiology 2020;10:1773733.

2.Caramelo F, Ferreira N, Oliveiros B. Estimation of risk factors for COVID-19 mortalitypreliminary results. MedRxiv 2020

3.Bhagavathula AS, Rahmani J, Aldhaleei WA, Kumar P, Rovetta A. Global, Regional and National Incidence and Case-fatality rates of Novel Coronavirus (COVID-19) across 154 countries and territories: A systematic assessment of cases reported from January to March 16, 2020. medRxiv 2020

4.Onder G, Rezza G, Brusaferro S. Case-fatality rate and characteristics of patients dying in relation to COVID-19 in Italy. Jama 2020

5.Nixon J, Ulmann P. The relationship between health care expenditure and health outcomes. The European Journal of Health Economics 2006;7:7-18.

6.Novignon J, Olakojo SA, Nonvignon J. The effects of public and private health care expenditure on health status in sub-Saharan Africa: new evidence from panel data analysis. Health economics review 2012;2:22.

7.Bradley EH, Elkins BR, Herrin J, Elbel B. Health and social services expenditures: associations with health outcomes. BMJ Qual saf 2011;20:826-831.

8.Jebeli SSH, Hadian M, Souresrafil A. Study of health resource and health outcomes: Organization of economic corporation and development panel data analysis. Journal of education and health promotion 2019;8

9.Farahani M, Price N, El-Halabi S, Mlaudzi N, Keapoletswe K, Lebelonyane R, et al. Impact of health system inputs on health outcome: a multilevel longitudinal analysis of Botswana National Antiretroviral Program (2002-2013). PloS one 2016;11

10.Xesfingi S, Vozikis A. Patient satisfaction with the healthcare system: assessing the impact of socio-economic and healthcare provision factors. BMC health services research 2016;16:94.

11.Triche TJ, Laird PW, Siegmund KD. Beta regression improves the detection of differential DNA methylation for epigenetic epidemiology. BioRxiv 2016:054643.

12.McMurray A, Pearson T, Casarim F. Guidance on applying the Monte Carlo approach to uncertainty analyses in forestry and greenhouse gas accounting. Winrock International 2017

13. Onder G, Rezza G, Brusaferro S. Case-Fatality Rate and Characteristics of Patients Dying in Relation to COVID-19 in Italy. JAMA. 2020;323(18):1775-6

14.Fok ML-Y, Hayes RD, Chang C-K, Stewart R, Callard FJ, Moran P. Life expectancy at birth and all-cause mortality among people with personality disorder. Journal of psychosomatic research 2012;73:104-107.

15.Bank W. Hospital beds (per 1,000 people) [cited 2020/12/5 2020/12/5]. Available from: https://data.worldbank.org/indicator/SH.MED.BEDS.ZS.

16.Bank W. Current health expenditure per capita, PPP (current international \$) 2020/12/05]. Available from: https://data.worldbank.org/indicator/SH.XPD.CHEX.PP.CD.

17.Bank W. Population ages 65 and above (\% of total population) [cited 2020 2020/12/05]. Available from: https://data.worldbank.org/indicator/SP.POP.65UP.TO.ZS. 


\section{Figures}

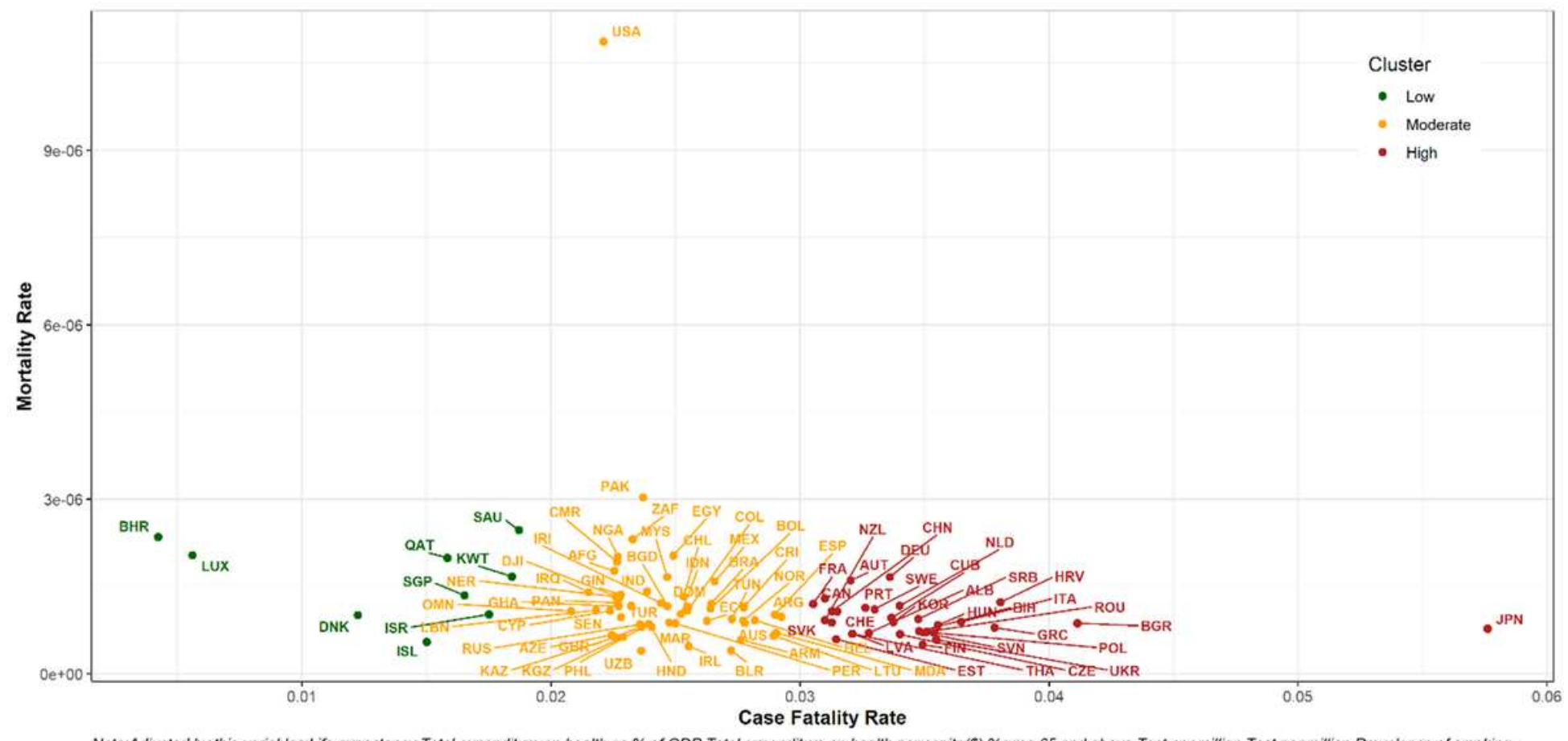

Note:Adjusted by this variables:Life expectancy.Total expenditure on health as \% of GDP, Total expenditure on health per capita(\$),\%ages 65 and above, Test per million, Test per million,Prevalence of smoking, Prevalence of Diabetes Mellitus, Nurse(per 1000 people, Physician(per 1000 people) and Hospital beds(per 1000 people)

\section{Figure 1}

Country-based clustering due to the low, medium and high rate of adjusted mortality rate and case fatality rate. The Monte Carlo simulation performed on the predicted values for each country, showed that the adjusted mortality rate and case fatality rate for the world were 0.000001 and 0.026 , respectively. 